\title{
Magnetic moment of a slab of type-I superconductor: Theoretical model and experiment
}

\author{
V. Kozhevnikov ${ }^{1}$ and C. Van Haesendonck ${ }^{2}$ \\ ${ }^{1}$ Tulsa Community College, Tulsa, Oklahoma 74119, USA \\ ${ }^{2}$ Solid State Physics and Magnetism Section, KU Leuven, BE-3001 Leuven, Belgium
}

(Received 24 July 2014; revised manuscript received 14 September 2014; published 30 September 2014)

\begin{abstract}
The magnetic moment $M$ of superconducting samples with a nonzero demagnetizing factor is one of the most important but not always completely understood sample characteristics. Here we calculate the magnetic moment of a slab of type-I superconductor in an arbitrarily oriented magnetic field, applying a recently developed model of the intermediate state (IS) [V. Kozhevnikov et al., Phys. Rev. B 89, 100503(R) (2014)]. We introduce and employ a novel form of the thermodynamic potential, which allows us to derive a general formula for the total free energy of the slab. The magnetic moment, calculated from the total free energy, is consistent with experimental data reported in this paper as well as with data available in the literature. Also, the calculated moment solves a long-standing puzzle of experimental $M(H)$ curves ( $H$ is the applied field) and provides insight in the field driven evolution of the magnetic flux density in the domain structure of the IS. This paper completes our presentation of a theoretical model which consistently addresses all properties of the IS, thus solving the problem of the IS in a slab that was put forward by Landau almost eight decades ago.
\end{abstract}

DOI: 10.1103/PhysRevB.90.104519

PACS number(s): 74.20.-z, 74.25.Ha

\section{INTRODUCTION}

The magnetic moment $M$ induced in a sample by a dc magnetic field $H$ is a hallmark of superconductivity, providing access to the main asset of superconductivity, i.e., the condensation energy. At the transition to the normal (N) state, the magnetic energy of a singly connected sample, $-\int \mathbf{M} \cdot d \mathbf{H}$, becomes equal to its condensation energy $\left(H_{c}^{2} / 8 \pi\right) V$, where $H_{c}$ is the thermodynamic critical field and $V$ is the sample volume [1]. This implies that the area under a graph of the reduced moment $4 \pi M / V H_{c}$ versus the reduced field $H / H_{c}$ has to be equal to $1 / 2$, regardless of the sample material and the shape, as soon as $\mathbf{M}$ is aligned (antiparallel) with $\mathbf{H}$ (below we will refer to this condition as the "rule of $1 / 2$ "). The magnetic moment of a superconductor (long rod in a parallel field) was measured for the first time by Ryabinin and Shubnikov in 1934 [2], constituting "the clearest confirmation of the Meissner effect" [3]. Nowadays the magnetic moment is probably the most often measured quantity in studies of superconductivity.

The behavior of $\mathbf{M}(\mathbf{H})$ is well understood for superconductors with a zero demagnetizing factor $\eta$, i.e., for long cylinders or slabs in a parallel field [1]. However, this is not always the case for other sample shapes or other field orientations, in particular for the practically important case of a slab in a perpendicular field. Available theoretical analyses of $M(H)$ for this case for both type-I and type-II superconductors (see, e.g., Refs. [4-7]) are either incomplete or questionable. This prevents one from using the rich information contained in the $M(H)$ curves to its full extent.

In this paper we focus on the magnetic moment of an infinite slab (samples with an aspect ratio $\gtrsim 10$ ) of a typeI superconductor in an arbitrarily oriented magnetic field. Unless the field is parallel to the sample, the sample will be in the intermediate state (IS) over the full range of the superconducting state, from $H=0$ up to $H_{c i}$, which is the critical field of the IS-N transition.

The problem of the IS was outlined for the first time by Gorter and Casimir in their first treatment of superconductivity as a new thermodynamic state of matter [8]. Bulk properties of superconductors in the IS, including the magnetic moment, were addressed theoretically by F. London [9] and Peierls [10]. The domain structure of the IS in an infinite slab was considered for the first time by Landau [11]. A brief historical overview of the studies of the IS was presented in Ref. [12], where we introduced a model of the IS for a slab in a tilted field. Our model consistently addresses the period $D$ of the laminar flux structure, the fractions $\rho_{n}$ and $\rho_{s}\left(=1-\rho_{n}\right)$ of the $\mathrm{N}$ component and the superconducting (S) component, respectively, the critical field $H_{c i}$ of the $\mathrm{S}-\mathrm{N}$ transition, and the induction $B$ in the $\mathrm{N}$ domains. Below we will apply this model to calculate the magnetic moment. It will be shown that the magnetic moment calculated from the correctly chosen thermodynamic potential solves the long-standing puzzle of an "excess" moment that appears in the $M(H)$ curves, and provides information about the induction $B$ in the normal domains and its evolution with the applied field.

\section{MAGNETIC MOMENT OF A LONG CYLINDER}

First, we consider the magnetic moment of a long cylinder of a type-I superconductor of volume $V$ and length $l \gg \sqrt{A}$, where $A=V / l$ is the cross sectional area of the cylinder, in a parallel field $H$, implying that $\eta=0$. The cylinder is in the Meissner state and its magnetic moment is $M=I A / c$, where $c$ is the speed of light. The screening current $I=g l$, where $g=\Delta B c / 4 \pi=c H / 4 \pi$ is the linear density of the current and the jump $\Delta B$ at the sample boundary is equal to the external field $H$. Taking into account the direction of $\mathbf{g}$, one arrives at the familiar formula [13]

$$
M=-\left(\frac{c H}{4 \pi} l\right) \frac{A}{c}=-\frac{H}{4 \pi} V,
$$

where the negative sign implies that $\mathbf{M}$ is directed opposite to $\mathbf{H}$.

On the other hand, $M$ can be calculated from an appropriately constructed thermodynamic potential $\widetilde{F}_{M}(T, V, H)$, referred to as the total free energy of the sample for which 
$\mathbf{M}=-\nabla_{\mathbf{H}} \widetilde{F}_{M}$ [13]. For samples with cylindrical geometry and negligible demagnetizing and magnetostriction effects the total free energy is [5]

$$
\widetilde{F}_{M}=\widetilde{F}+\frac{H^{2} V}{8 \pi},
$$

where $\widetilde{F}=\widetilde{F}(T, V, H)$ is another form of the free energy, often referred to as the Gibbs potential, which is the Legendre transform of the Helmholtz free energy $F(T, V, B)[5,13,14]$,

$$
\widetilde{F}=F-\frac{B H}{4 \pi} V .
$$

Note that (a) the addition of $H^{2} V / 8 \pi$ (energy of the applied field if the sample is absent) in Eq. (2) does not affect the differential thermodynamic relationships and hence also does not affect the thermodynamic properties of the sample, but the addition makes it possible to calculate the moment induced by the magnetic field [13]; (b) the difference between $\widetilde{F}$ and $F$ is associated with the work done by the magnet power supply when the magnetic flux in the system changes, e.g., at the transition of the sample from the superconducting (S) to the $\mathrm{N}$ state. If the flux is fixed, which is the case for a slab in perpendicular field, $\widetilde{F}=F[13,14]$.

For our sample in the Meissner state one obtains that

$$
\widetilde{F}_{M}=F_{n}-\frac{H_{c}^{2}}{8 \pi} V+\frac{H^{2}}{8 \pi} V=F_{s 0}+\frac{H^{2}}{8 \pi} V,
$$

where $F_{n}$ is the Helmholtz free energy in the $\mathrm{N}$ state and $F_{s 0}$ is the free energy in the $\mathrm{S}$ state at zero field. Then one obtains for $M$ the same expression as that given by Eq. (1),

$$
M \equiv-\frac{d \widetilde{F}_{M}}{d H}=-\frac{H}{4 \pi} V .
$$

We conclude that electrodynamics and thermodynamics yield identical results for the magnetic moment of a sample with a zero demagnetizing factor or when inhomogeneities of the field and current at the sample edges are neglected.

Now we turn the cylinder so that $H$ is perpendicular to its axis. Then, according to the standard theory $[9,10]$, the cylinder is in the Meissner state with $4 \pi M=-H V /(1-\eta)$ until the field reaches $H_{i}=H_{c}(1-\eta)$, where the demagnetizing factor for the cylinder $\eta=1 / 2$. For the field range $H_{c}(1-\eta)<H \leqslant$ $H_{c}$ the cylinder is in the IS where the average induction $\bar{B}=$ $H / \eta-H_{c}(1-\eta) / \eta$ and the magnetic moment $4 \pi M / V=$ $-\left(H_{c}-H\right) / \eta[4,5,13]$. Graphs for these quantities versus $H$ are presented in Fig. 1.

Already in early studies (see Refs. $[4,13]$ for references) it has been revealed that (a) the transition from the Meissner state to the IS occurs at $H_{i}>(1-\eta) H_{c}$, and (b) the IS-N transition takes place at $H_{c i}<H_{c}$. The magnetization in the IS was measured for the first time by Desirant and Shoenberg [16]. The samples, which were measured in a perpendicular field, were tin and mercury cylinders with diameters from 0.034 to $0.36 \mathrm{~mm}$. It was found that the difference between $H_{i}$ and $0.5 H_{c}$ and between $H_{c i}$ and $H_{c}$ lies in the range from about $5 \%$ to $20 \%$, depending on the diameter of the cylinders: The smaller the diameter, the larger is the difference. On the other hand, the fact that $H_{i}$ is larger than $(1-\eta) H_{c}$ implies that the
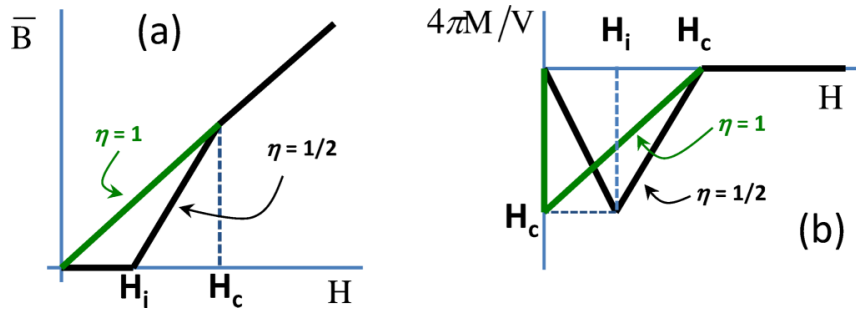

FIG. 1. (Color online) Average magnetic induction in (a) and the moment of (b) an infinite slab $(\eta=1)$ and a cylinder $(\eta=1 / 2)$ in a perpendicular field $H$, as expected from the standard theory.

magnitude of $4 \pi M / V$ at $H=H_{i}$ exceeds $H_{c}$. This "excess" moment is also clearly seen in the data presented in Ref. [16].

The reduction of $H_{c i}$ has been discussed in Refs. [1,14] and addressed in detail in Ref. [12]: The reduction is dictated by thermodynamics due to the price paid by the sample for inhomogeneities of the field and domains near the sample surface and for the $\mathrm{S} / \mathrm{N}$ interfaces in the bulk of the sample. However, the origin of the excess moment for which $\left|4 \pi M\left(H_{i}\right) / V\right|>H_{c}$ (although it is consistent with the rule of $1 / 2$ ) has remained a puzzle since in all theories this quantity is equal to $H_{c}$. In particular, in the Landau laminar model (LLM) [6] the magnitude of $4 \pi M\left(H_{i}\right) / V$ is equal to the induction in the first $\mathrm{N}$ domain, which cannot be larger than $H_{c}$. This puzzle will be solved below.

\section{MAGNETIC MOMENT OF A SLAB: THEORY}

The schematics of our model of the IS is presented in Fig. 2. In this model the screening current in each $\mathrm{S}$ lamina consists of two components screening the perpendicular $\left(B_{\perp}\right)$ and parallel $\left(B_{\|}=H_{\|}\right)$components of the field around it. Corresponding currents are $I_{\perp}=g_{\perp} d$ and $I_{\|}=g_{\|} w$, where $d$ is the sample thickness (the length of the lamina in the $z$ direction) and $w$ is the sample width (the length of the lamina in the $y$ direction).

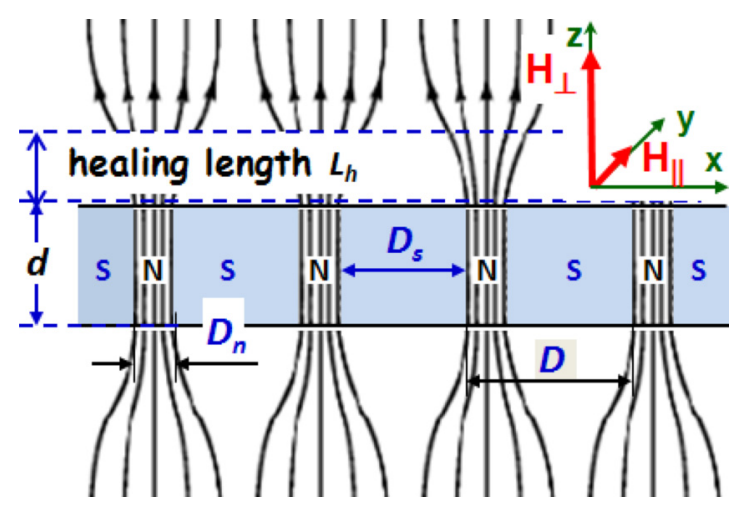

FIG. 2. (Color online) Cross sectional view of the modeled superconducting slab in the intermediate state. $H_{\perp}$ and $H_{\|}$are the perpendicular and parallel components of the applied field, respectively. Domains are rectangular parallelepipeds extended along $H_{\|}$. The healing length $L_{h}$ is the characteristic distance over which the field passing through the sample relaxes to its uniform state. The parallel component of the flux density $B_{\|}$in the $\mathrm{N}$ domains is equal to $H_{\|}$, while the perpendicular component $B_{\perp}=H_{\perp} \rho_{n}$, where $\rho_{n}=D_{n} / D$ is the fraction of the $\mathrm{N}$ phase. 
The current screening $B_{\perp}$ is $I_{\perp}=g_{\perp} d=c B_{\perp} d / 4 \pi$ and the current screening $B_{\|}$is $I_{\|}=g_{\|} w=c B_{\|} w / 4 \pi$. Therefore, for a sample of length $L$ (the length in the $x$ direction) containing $\aleph=L / D$ laminae, one obtains

$$
\begin{aligned}
M_{\perp} & =\frac{I_{\perp} A_{\perp}}{c} \aleph=-\frac{c B_{\perp} d}{4 \pi} \frac{D_{s} w}{c} \frac{L}{D}=-\frac{B_{\perp} \rho_{s} V}{4 \pi} \\
& =-\frac{B_{\perp}}{4 \pi}\left(1-\rho_{n}\right) V=-\frac{H_{c}}{4 \pi}\left(b_{\perp}-h_{\perp}\right) V
\end{aligned}
$$

and

$$
\begin{aligned}
M_{\|} & =\frac{I_{\|} A_{\|}}{c} \aleph=-\frac{c B_{\|} w}{4 \pi} \frac{D_{s} d}{c} \frac{L}{D}=-\frac{B_{\|} \rho_{s} V}{4 \pi} \\
& =-\frac{B_{\|}}{4 \pi}\left(1-\rho_{n}\right) V=-\frac{H_{c}}{4 \pi}\left(b_{\perp}-h_{\perp}\right) \frac{h_{\|}}{b_{\perp}} V,
\end{aligned}
$$

where $D_{s}$ is the width of the $\mathrm{S}$ laminae and all other notations are the same as in Ref. [12]; the small symbols $b$ and $h$ refer to the reduced flux density $B / H_{c}$ and the reduced applied field $H / H_{c}$, respectively. It is accounted that the flux of $H_{\perp}$ is fixed and therefore $B_{\perp} \rho_{n}=H_{\perp}$ [12]. A minus sign in front of $M_{\perp}$ and $M_{\|}$means that they are directed opposite to the corresponding components of the magnetic field.

The magnitude of the moment $M^{2}=M_{\|}^{2}+M_{\perp}^{2}$ is

$$
\begin{aligned}
M^{2} & =\left(\frac{V H_{c}}{4 \pi}\right)^{2}\left[b_{\perp}^{2}\left(1-\frac{h_{\perp}}{b_{\perp}}\right)^{2}+h_{\|}^{2}\left(1-\frac{h_{\perp}}{b_{\perp}}\right)^{2}\right] \\
& =\left[\frac{V H_{c}}{4 \pi}\left(1-\rho_{n}\right) b\right]^{2}=\left[\frac{V}{4 \pi}\left(1-\rho_{n}\right) B\right]^{2},
\end{aligned}
$$

and the moment $\mathbf{M}$ is

$$
4 \pi \mathbf{M}=-V\left(1-\rho_{n}\right) \mathbf{B} .
$$

Note that the moment is opposite to the induction in the laminae and does not make a $180^{\circ}$ angle with the applied tilted field $\mathbf{H}$. The angle between $\mathbf{M}$ and $\mathbf{H}$ changes with the magnitude of $\mathbf{H}$, thus making dc measurements of the moment in a tilted field meaningless.

Now, if $H_{\perp} \rightarrow 0$, the sample approaches the Meissner state where $M_{\|}$[Eq. (7)] converts to $M$ as in Eqs. (1) and (5). If $H_{\|}=0$ and the induction $B$ in the $\mathrm{N}$ domains is $H_{c}$ (very thick samples [17]), the moment becomes the same as that in the standard theory and in the LLM [6]: $4 \pi M / V=H-H_{c}$ and $H_{c i}=H_{c}$. Consequently, Eq. (9) meets the mandatory "rule of $1 / 2$ " in the case of a thick sample. At the IS/N transition, $M=$ 0 , implying that $\rho_{n}=1$. Thus the condition $M=0$ is identical to the condition $\rho_{n}=1$ from which $H_{c i}$ was calculated in Ref. [12].

However, in real samples $H_{c i}$ is less than $H_{c}[1,12,14,18]$. Then, taking into account that $B(H=0)=H_{c}$ [12] and that the experimentally obtained $\rho_{n}(H)$ dependence is linear $[4,12,18,19]$, one finds that Eq. (9) violates the rule of $1 / 2$. Therefore one can expect that Eq. (9) is valid for thick samples only. We note that the condition needed to have a thick sample is identical to the condition of negligibly small effects associated with the field and domain inhomogeneities near the sample surface [12]; it is exactly under this condition that the correct result for a long rod was obtained from electrodynamics in the previous section.
Now we turn to thermodynamics. The important advantage of thermodynamics is that it allows one to calculate $M$ even if the specific arrangement of the currents is not known [5]. The free energy $\widetilde{F}$ for a slab in a tilted field was calculated in Ref. [12] using the simplified approximation introduced by Tinkham for the field distribution near the sample surface. In this approximation the field $B_{\perp}$ exiting the sample relaxes to the uniform applied field $H_{\perp}$ over the "healing length" $L_{h}$. The latter is calculated to be $L_{h}^{-1}=D_{n}^{-1}+D_{s}^{-1}$, where $D_{n}$ and $D_{s}$ are the widths of the $\mathrm{N}$ and $\mathrm{S}$ laminae, respectively, as illustrated in Fig. 1 . The potential $\widetilde{f} \equiv \widetilde{F} / V$ has the form

$$
\tilde{f}=f_{n}+\frac{H_{c}^{2}}{8 \pi}\left[\rho_{n}-1+\frac{h_{\perp}^{2}}{\rho_{n}}-\rho_{n} h_{\|}^{2}+4 h_{\perp} \sqrt{\frac{\delta}{d}}\left(1-\rho_{n}\right)\right],
$$

where $\delta$ is the domain wall parameter, characterizing the width of the transition region between the $\mathrm{N}$ and $\mathrm{S}$ domains [1], $d$ is the sample thickness, and $f_{n}=F_{n} / V$. Now we claim that the appropriate form of $\widetilde{F}_{M}$ is

$$
\widetilde{F}_{M}=\widetilde{F}+\left[\frac{H_{\|}^{2}}{8 \pi}-\frac{H_{\perp}^{2}}{8 \pi}\right] V .
$$

Similar as in Eq. (2), the term $H_{\|}^{2} / 8 \pi$ is needed to calculate the magnetic moment induced by the longitudinal component of the field $H_{\|}$, and the term $-H_{\perp}^{2} / 8 \pi$ is needed to calculate the moment induced by the transverse field component $H_{\perp}$. Surprisingly, the latter term is negative. This is due to the demagnetizing field inside the $\mathrm{N}$ laminae which is absent for the above considered long cylinder in a parallel field. The explanation for the appearance of these two terms is given in the Appendix.

Now, plugging $\rho_{n}$ [see Eq. (5) in Ref. [12]] into Eq. (10) and taking into account the expression for $b_{\perp}$ [see Eq. (7) in Ref. [12]] reading that

$$
b_{\perp}^{2}=1-4 h_{\perp} \sqrt{\delta / d}-h_{\|}^{2},
$$

we arrive at a compact expression for $\widetilde{f}_{M}\left(\equiv \widetilde{F}_{M} / V\right)$ that closely resembles the canonical form of the total free energy for magnetic materials [13]:

$$
\widetilde{f}_{M}=f_{n}-\frac{H_{c}^{2}}{8 \pi}\left(b_{\perp}-h_{\perp}\right)^{2}=f_{n}-\frac{B_{\perp}^{2}}{8 \pi}\left(1-\rho_{n}\right)^{2} .
$$

Before continuing we take a closer look at Eq. (13). First, we note that $\widetilde{f}_{M}$ depends only on the perpendicular components of the field inside the sample $\left(b_{\perp}\right)$ and of the applied field $\left(h_{\perp}\right)$. However, this does not imply that $\widetilde{f}_{M}$ is independent of $h_{\|}$since $b_{\perp}$ depends on $h_{\|}$[Eq. (12)]. We then see that $\widetilde{f}_{M}$ in the superconducting state is always smaller than $\widetilde{f}_{M}$ in the normal state (where it is equal to $f_{n}$ ), as it should be. At the IS-N transition the field $B$ inside the sample becomes equal to the applied field $H$ and the fraction $\rho_{n}$ becomes equal to unity. In Eq. (13) both these conditions lead to $\widetilde{f}_{M}(I S)=\widetilde{f}_{M}(N)$, as it should be at the IS-N transition. Therefore $\widetilde{F}_{M}$ possesses the characteristics necessary for the total free energy. The final characteristic is the magnetic moment and we consider it now. 
Let us introduce the unit vectors along the $y$ and $z$ axes: $\hat{\mathbf{y}}$ and $\hat{\mathbf{z}}$ (see Fig. 1). Then we expect that

$$
\mathbf{M}=-\left(\frac{\partial \widetilde{F}_{M}}{\partial H_{\|}} \hat{\mathbf{y}}+\frac{\partial \widetilde{F}_{M}}{\partial H_{\perp}} \hat{\mathbf{z}}\right) .
$$

For the parallel component we have

$$
\frac{\partial \widetilde{F}_{M}}{\partial H_{\|}}=\frac{V}{H_{c}} \frac{\partial \widetilde{f}_{M}}{\partial h_{\|}}=\frac{V}{H_{c}} \frac{H_{c}^{2}}{8 \pi} 2\left(b_{\perp}-h_{\perp}\right) \frac{\partial b_{\perp}}{\partial h_{\|}} .
$$

Using Eq. (12) we find that $\partial b_{\perp} / \partial h_{\|}=h_{\|} / b_{\perp}$ (note that this derivative does not depend on the specific form of the approximation for the field distribution near the surface) and therefore

$$
\begin{aligned}
\frac{\partial \widetilde{F}_{M}}{\partial H_{\|}} & =\frac{V}{H_{c}} \frac{H_{c}^{2}}{8 \pi} 2\left(b_{\perp}-h_{\perp}\right) \frac{h_{\|}}{b_{\perp}} \\
& =\frac{V H_{c}}{4 \pi}\left(1-\frac{h_{\perp}}{b_{\perp}}\right) h_{\|}=\frac{V}{4 \pi}\left(1-\rho_{n}\right) H_{\|} .
\end{aligned}
$$

This is the same as the result for $M_{\|}$in Eq. (7) above.

For the perpendicular component we have

$$
\begin{aligned}
\frac{\partial \widetilde{F}_{M}}{\partial H_{\perp}} & =\frac{V}{H_{c}} \frac{\partial \widetilde{f}_{M}}{\partial h_{\perp}}=-\frac{V}{H_{c}} \frac{2 H_{c}^{2}}{8 \pi}\left(b_{\perp}-h_{\perp}\right)\left(\frac{\partial b_{\perp}}{\partial h_{\perp}}-1\right) \\
& =\frac{V}{4 \pi}\left(1-\rho_{n}\right)\left(1-\frac{\partial B_{\perp}}{\partial H_{\perp}}\right) B_{\perp} .
\end{aligned}
$$

This is the same as $M_{\perp}$ in Eq. (6) provided that $\left|\partial b_{\perp} / \partial h_{\perp}\right| \ll$ 1 , as is the case for thick samples where $B$ is fixed $\left(=H_{c}\right)$. This confirms our expectation that $M$ in Eq. (9) represents the magnetic moment in the limit of very thick samples. On the other hand, Eq. (17) accounts for the behavior of the moment in real samples. The thinner the sample, the smaller is $H_{c i}$ and the larger is $\left|\partial b_{\perp} / \partial h_{\perp}\right|$ (this derivative is negative) [12] and therefore the larger is the magnitude of $M_{\perp}=-\partial \tilde{F}_{M} / \partial H_{\perp}$. In particular, in a perpendicular field $|4 \pi M(H \rightarrow 0) / V|$ exceeds $H_{c}$ due to the presence of the factor $(1-\partial B / \partial H)$. This explains how the system meets the rule of $1 / 2$ in the intermediate state and why the magnitude of the magnetic moment at $H_{i}$ is larger than $H_{c} V / 4 \pi$ : because the flux density in the $\mathrm{N}$ domains decreases with the applied field [12], implying that the $\mathrm{N}$ phase can be stable at fields lower than $H_{c}$, as it follows from our model and is confirmed by the experiment [12].

We now check what happens if $H_{\perp} \rightarrow 0$ in the presence of a field component $H_{\|}$. In that case the sample approaches the Meissner state and $\widetilde{F}_{M}$ becomes the same as for a parallel field in Eq. (4):

$$
\widetilde{F}_{M} \rightarrow F_{n}-V H_{c}^{2}\left(1-h_{\|}^{2}\right) / 8 \pi=F_{s 0}+V H^{2} / 8 \pi .
$$

We conclude that the potential $\widetilde{F}_{M}$ in Eq. (13) is the total free energy of an infinite slab of a type-I superconductor regardless of the orientation of the magnetic field: parallel, tilted, or perpendicular. It is important to note that $\widetilde{F}_{M}$ in Eq. (13) is independent of the specific approximation for the energy term associated with the field and laminae inhomogeneity near the surface. Therefore Eq. (13) is more general than Eq. (10). It is also important that the form of $\widetilde{F}_{M}$ [Eq. (11)] was chosen based on the consideration of the demagnetizing effects, which do not depend on the sample material. Therefore Eq. (11) is the general form of the total free energy of any (type-I and type-II) superconducting slab. As for now, we leave open the question concerning the applicability of Eq. (13) to type-II superconductors, because the answer requires knowledge of the magnetic structure of the vortex core and its evolution with the applied field. We note that, being a fundamental and very interesting problem on its own, the knowledge of the vortex core structure is also necessary for the extraction of the microscopic parameters from a muon spin rotation $(\mu \mathrm{SR})$ spectrum [20].

To conclude this section we introduce one more (the most canonical) form of the total free energy of a superconducting slab in addition to the free energy given by Eq. (13),

$$
\widetilde{F}_{M}=\widetilde{F}_{M}(H=0)-\int \mathbf{M} \cdot d \mathbf{H}=F_{s 0}-\int \mathbf{M} \cdot d \mathbf{H},
$$

where the components of $\mathbf{M}$ are given by Eqs. (16) and (17).

\section{MAGNETIC MOMENT OF A SLAB: EXPERIMENT}

The main challenge in measuring the moment and other equilibrium magnetic properties in the IS is to fulfill the requirement to have a singly connected sample. For real samples this means the absence of pinning, since pinning centers act as holes trapping the flux. Because the typical sample size for measuring dc magnetization is rather big $(\approx 4-6 \mathrm{~mm})$, the magnetic flux is almost inevitably trapped near $H=0$ both at increasing and decreasing fields. Then the magnetic moment arising from the current(s) protecting the trapped flux can significantly exceed the diamagnetic sample response, thus strongly or even completely overshadowing the signal of interest, and often producing "hornlike" shaped $M(H)$ curves, as discussed in Ref. [4] and shown, as an example, in Fig. 3. In dirty samples the equilibrium response is either not visible at all or is seen within a small range of $H$ near $H_{c i}$. The cleaner the sample, the wider is this range.

Experimental data for the magnetic moment measured in perpendicular field for two high-purity indium films of thickness $3.86 \mu \mathrm{m}$ (sample In-A) and $2.79 \mu \mathrm{m}$ (In-B) are presented in Figs. 4 and 5, respectively. The choice of indium

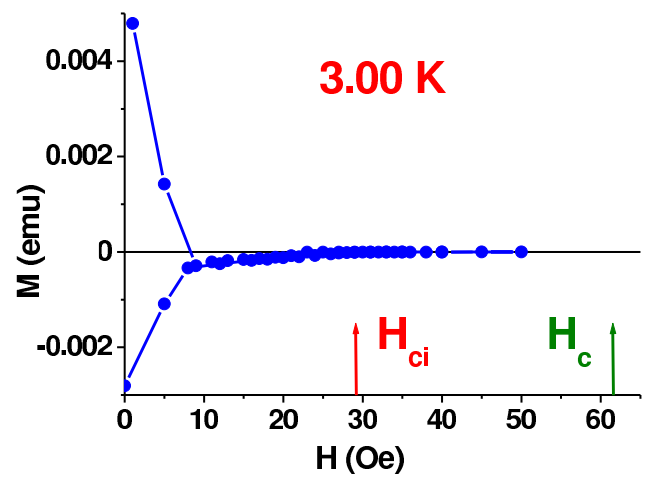

FIG. 3. (Color online) Magnetic moment of the In-B sample measured in a perpendicular field at $3 \mathrm{~K}$. Relevant data are above $10 \mathrm{Oe}$; at lower fields the moment is due to the current protecting the flux trapped at pinning centers. $H_{c}$ is the thermodynamic critical field and $H_{c i}$ is the critical field of the transition from the intermediate to the normal state. 


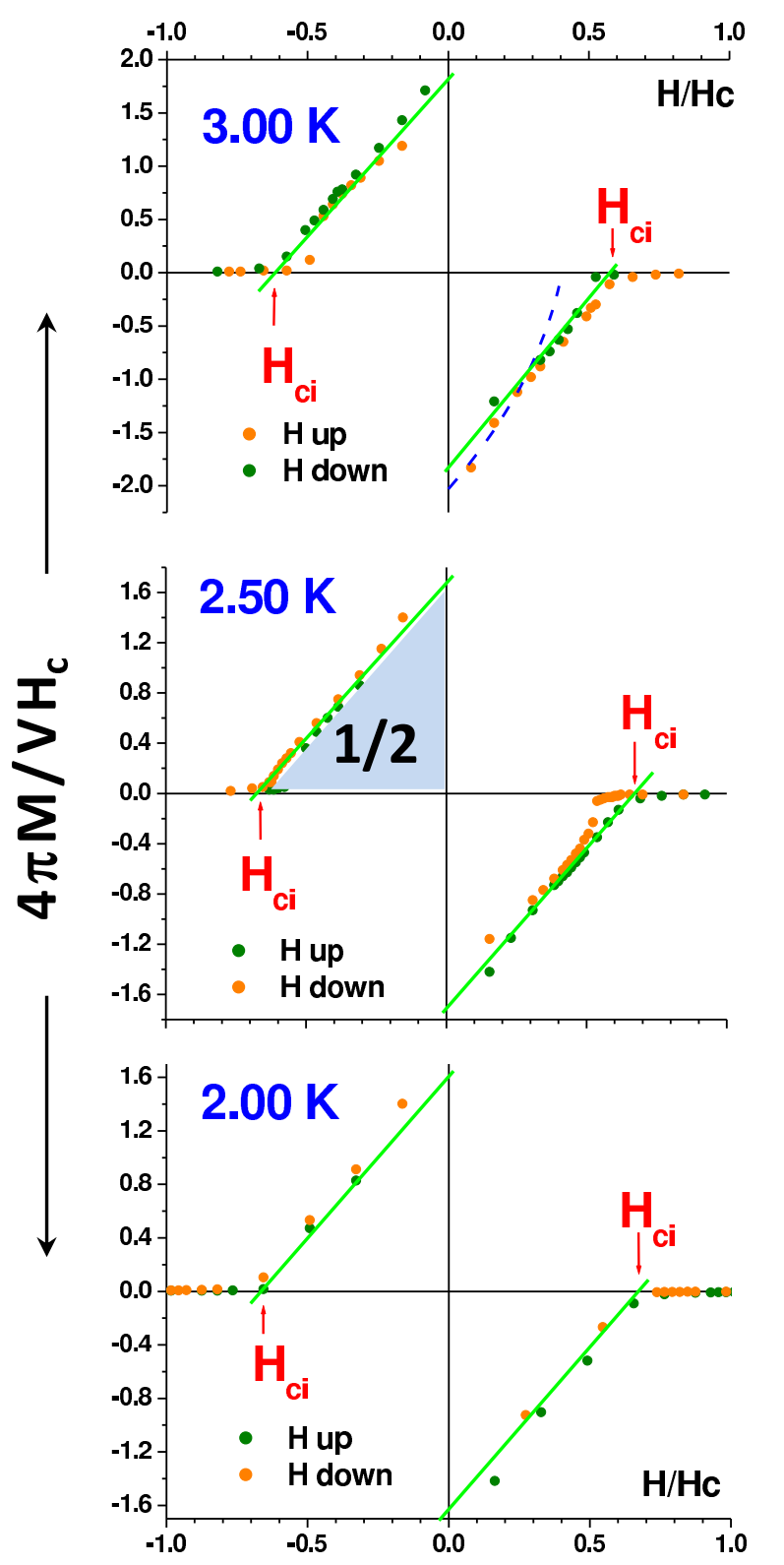

FIG. 4. (Color online) Magnetic moment of the $3.86 \mu \mathrm{m}$ thick indium film sample (In-A) in a perpendicular field at the different temperatures indicated on the graphs. The dashed curve in the graph for $3 \mathrm{~K}$ represents the moment inferred from our model with $\delta$ calculated using the Pippard coherence length $\xi_{0}=380 \mathrm{~nm}$, as described in Ref. [12].

is motivated by the exceptional properties of the indium films. The surface of these films consists of nearly atomically flat terraces [21], resulting in a significant degree of specular reflection of electrons at the surface. Owing to that, the mean free path of the electrons can become very large, exceeding the film thickness. On the other hand, indium is an "archetypical" type-I material: Its Ginzburg-Landau parameter $\kappa(0.07)$ is one of the smallest (the second after aluminum) among the available superconductors. Therefore, In possesses a large $\mathrm{S} / \mathrm{N}$ interface energy, and hence a strong force driving the flux pattern to its equilibrium state. These two properties make indium the metal of choice for studies of the equilibrium

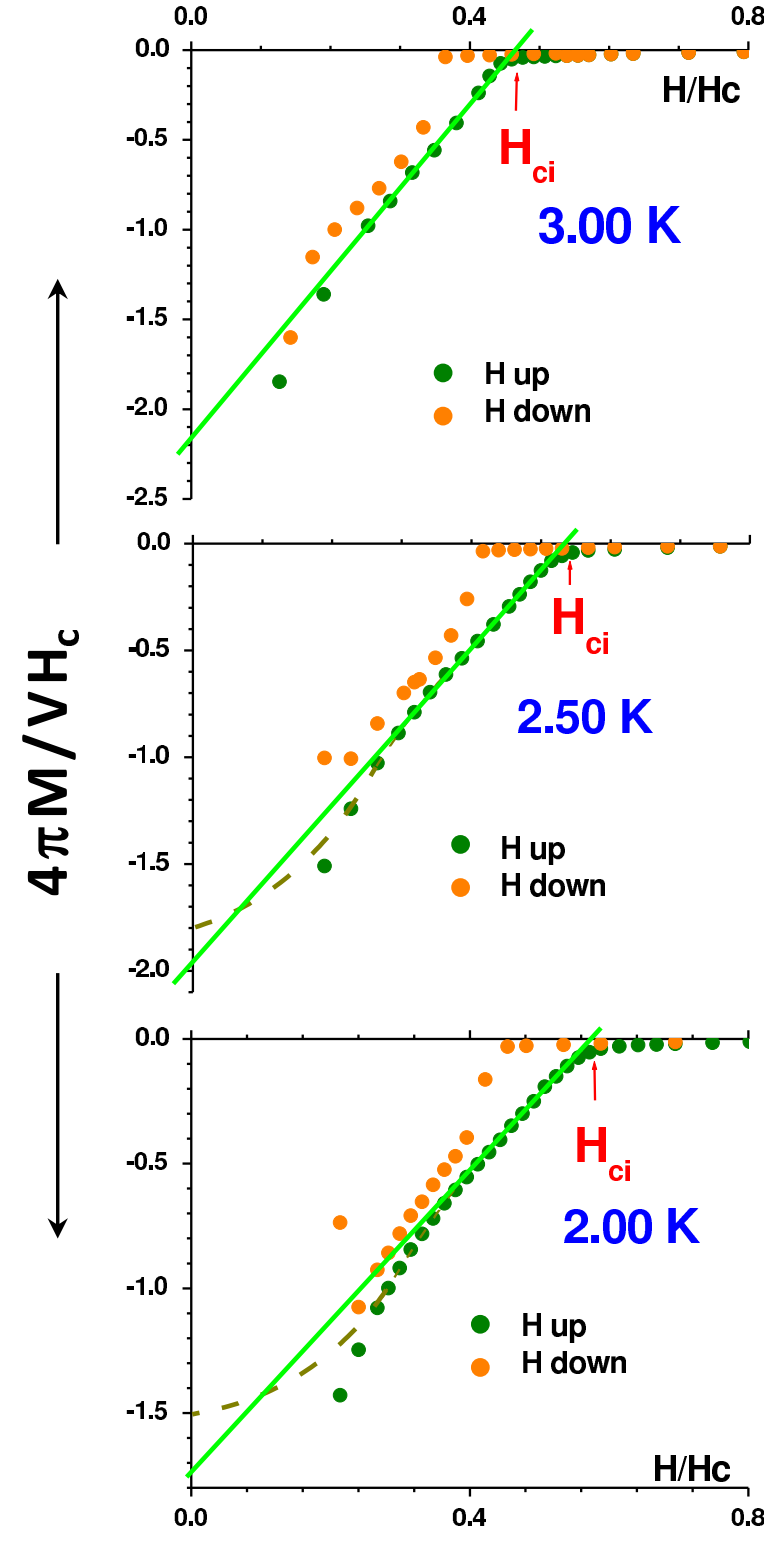

FIG. 5. (Color online) Magnetic moment of the $2.79 \mu \mathrm{m}$ thick indium film sample (In-B) in a perpendicular field at the different indicated temperatures. The dashed curves are extrapolations of the experimental data consistent with the rule of $1 / 2$.

properties of type-I superconductors (see Ref. [12] for more details).

The film thickness was measured with an optical interference profiler (Taylor Hobson CCI MP system), reducing the uncertainty to less than $0.1 \%$. The residual resistivity ratio of sample In-A is 610 , while for sample In-B the ratio is 480. Correspondingly, the mean free path is $12 \mu \mathrm{m}(\mathrm{In}-\mathrm{A})$ and $9 \mu \mathrm{m}$ (In-B). Volumes of the samples were determined from the slope of the $M(H)$ curves in the Meissner state measured in a parallel field and presented in Fig. 6. The volume of the In-A sample is $(9.79 \pm 0.08) \times 10^{-5} \mathrm{~cm}^{3}$ and the volume of the In-B sample is $(3.65 \pm 0.03) \times 10^{-5} \mathrm{~cm}^{3}$. The $M(H)$ curves presented in Fig. 6 also provide the data required to determine the thermodynamic critical field $H_{c}$. Similar to our other indium samples (see Refs. [12,21]), the data on $H_{c}$ of the In-A 


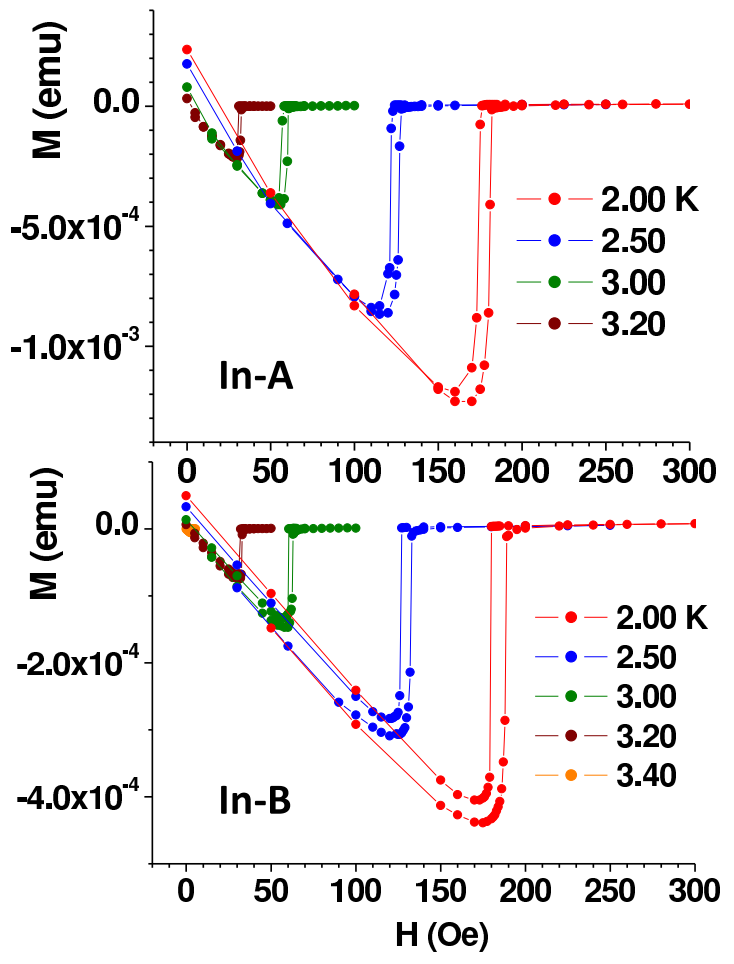

FIG. 6. (Color online) Magnetic moment of the In-A and In-B samples in a parallel field.

and In-B samples are in perfect agreement with the standard thermodynamic phase diagram of indium. All magnetization measurements were performed using a superconducting quantum interference device (SQUID) dc magnetometer [Quantum Design magnetic property measurement system (MPMS)]. Deep supercooling for a decreasing field, which can be seen in Figs. 4-6, confirms the high purity of the films as well as the fact that the S-N transitions in both parallel and perpendicular fields are first-order phase transitions.

When analyzing the graphs for the magnetic moment in perpendicular field (Figs. 4 and 5), we first note that the graphs meet the rule of $1 / 2$ : The area under the straight (green) line for all these graphs equals $1 / 2$ with an error $\lesssim 3 \%$. Therefore the obtained data represent the equilibrium moment. As can be seen from these graphs, the excess moment $4 \pi M / V H_{c}-1$ for $H \rightarrow 0$ increases with increasing temperature and with decreasing thickness $d$. This is fully consistent with our model, where the excess moment $\left(-\partial B_{\perp} / \partial H_{\perp}\right)$ is determined by the ratio $\delta / d$ [see Eq. (12)], and $\delta$ increases with temperature [12].

An interesting feature of these graphs is that the $M(H)$ dependence is close to linear within a large field range. As mentioned above, the linearity of $M(H)$ follows from the standard theory [see Fig. 1(b)] and from the LLM [6]; both are valid for thick samples. In such samples $B=H_{c}$ (fixed) and according to Eq. (9) the linearity of $M(H)$ is equivalent to the linear dependence of the normal fraction $\rho_{n}$ vs $H$. This is consistent with the available data that allows one to judge on the $\rho_{n}(H)$ dependence in the IS. These data include, in particular, results of electrical transport measurements with cylindrical wires in a perpendicular field $[4,19]$ and with film samples in a tilted field [12] (in these cases the flux pattern consists of ordered laminae perpendicular to the measuring current), and with results of $\mu \mathrm{SR}$ measurements for a slab in a perpendicular field [18]. It is worth noting that the linear law for $\rho_{n}(H)$ retrieved from the measurements is not trivial, especially for a slab in a perpendicular field, where the flux pattern becomes completely disordered [12].

However, in our samples the contribution of the surface related magnetic inhomogeneities is significant, as can be seen in Figs. 4 and 5 from the difference between $H_{c i}$ and $H_{c}$ and from the difference between $4 \pi M / V$ and $H_{c}$. Assuming that the linearity of $\rho(H)$ is a general property of the IS, the observed linearity of $M(H)$ in the In-A sample (Fig. 4) suggests that the decrease of $B$ for increasing fields is compensated by an increasing magnitude of the derivative $\partial B / \partial H$, making the product $B(1-\partial B / \partial H)$ a weak function of $H$. Qualitatively, this is consistent with Eq. (12), but our model is not supposed to address this issue quantitatively due to the oversimplified approximation for the near-surface field and domain inhomogeneities. An example of an $M(H)$ curve obtained using our model is added in Fig. 4 in the graph for $T=3 \mathrm{~K}$. The surface related contributions depend on $H$ and $\delta(T) / d$, and therefore it is unlikely that this compensation is universal. Indeed, the linearity of $M(H)$ breaks down at low fields, as can be seen in Fig. 5 in the graphs for 2 and $2.5 \mathrm{~K}$. The dashed lines qualitatively indicate the shape of the $M(H)$ curve following from the available data and the rule of $1 / 2$. This effect becomes more pronounced in the In-B sample (Fig. 5) because it is thinner than the In-A sample (Fig. 4).

Another intriguing feature seen in all of the graphs in Figs. 4 and 5 is the behavior of $M(H)$ near the critical field $H_{c i}$, where the graphs deviate from a straight line and smoothly approach the level $M=0$. Abnormal behavior of $B$ in this field region has also been revealed and stressed by Egorov et al. in the $\mu \mathrm{SR}$ study of the IS in a single-crystal tin slab [18]. The observed behavior of $M(H)$ near $H_{c i}$ may suggest that the IS-N transition is a second-order phase transition, as predicted by Landau [11,13]. However, the strong supercooling, which is clearly observed for decreasing fields in all graphs of Figs. 4 and 5 and is well documented in other sources $[4,12,18]$, does not leave any room for such an interpretation. The properties of superconductors near the IS-N transition constitute a fundamental problem, deserving a specially addressed investigation.

\section{SUMMARY}

A theoretical model for the intermediate state in an infinite slab introduced in Ref. [12] is applied for calculating the magnetic moment of the slab in an arbitrarily oriented magnetic field. A thermodynamic potential correctly accounting for the demagnetizing effects [Eq. (11)] and representing the total free energy of a superconducting slab is presented and used for the intermediate state of type-I superconductors. A general formula for the total free energy of a slab of a type-I superconductor [Eq. (13)] is derived and verified experimentally. It is demonstrated that this formula consistently addresses all equilibrium properties of a slab of a type-I superconductor in a magnetic field of arbitrary orientation, including the pure perpendicular and the pure parallel cases. Overall, the theoretical model presented in Ref. [12] and 
in this paper provides a comprehensive solution (albeit in a first-order approximation) of the problem of the intermediate state put forward by Landau in 1937. In particular, the model consistently explains the long known "abnormal" properties of superconductors in the intermediate state, including a reduction of the critical field and an excess magnetic moment, and overthrows the paradigm on the instability of the normal phase in type-I superconductors in a magnetic field below the thermodynamic critical field $H_{c}$ [12].

As indicated in Ref. [12], the limitations of our model are associated with an oversimplified approximation for the magnetic field distribution near the sample surface outside the sample and the neglect of the effect of rounded corners of the domain cross section near the surface inside the sample. Energy terms associated with these surface effects are responsible for all specific features of real samples, unless these samples are very thick. The features include a reduction of the induction in the normal domains when compared to $H_{c}$, a reduction of the critical field, an excess magnetic moment, the shape of the magnetization curves, etc. Similar surface effects are important for establishing equilibrium in type-II superconductors in the mixed state. The understanding and ability to model these surface related contributions is important for a proper interpretation of the properties of the real samples and for the extraction of microscopic parameters from the macroscopic properties, such as the $\mu$ SR spectrum. This problem has been addressed in a number of theoretical studies, e.g., Refs. [7,22], starting from the pioneering works of Landau [11,23]. However, none of the reported results pretend that they can be used for anything other than a qualitative or semiquantitative analysis (see, e.g., Fig. 5 in Ref. [12]). Solving this fundamental problem requires a targeted and complex experimental investigation.

\section{ACKNOWLEDGMENTS}

We thank Kelly Houben for help with the magnetization measurements and Luc Berckmoes (OCAS, Belgium) for help with measuring the film thicknesses. This work was supported by NSF (Grant No. DMR 0904157), by the Research Foundation-Flanders (FWO, Belgium), and by the Flemish Concerted Research Action (BOF KU Leuven, GOA/14/007) research program.

\section{APPENDIX}

The magnetic energy of a sample in the IS consists of surface and bulk terms. In our model [12] the bulk magnetic energy is the energy of the field $B$ in the $\mathrm{N}$ laminae $\left[V \rho_{n}\left(B_{\|}^{2}+B_{\perp}^{2}\right) / 8 \pi\right]$ plus $-V \rho_{n} H_{\|}^{2} / 4 \pi$, the term associated with the change of the flux of the parallel component of the applied field. Taking into account that the flux of $H_{\perp}$ is fixed $\left(B_{\perp} \rho_{n}=H_{\perp}\right)$, the sum of these contributions is
$V H_{\perp}^{2} / \rho_{n} 8 \pi-V \rho_{n} H_{\|}^{2} / 8 \pi$ [the third and fourth terms in Eq. (3) in Ref. [12]].

On the other hand, the bulk magnetic energy can be presented as the energy of interaction of the external field $H$ with the induced magnetic moment $M$, i.e., $E_{m b}=-\int \mathbf{M}$. $d \mathbf{H}=\int M_{\|} d H_{\|}+\int M_{\perp} d H_{\perp}$, where the effects associated with the field and domain distortion near the surface are neglected.

Consider an ellipsoidal superconducting sample in the IS placed in a magnetic field $H$ parallel to the sample axis of symmetry with demagnetizing factor $\eta$ with respect to this axis. For convenience we introduce a "magnetization" $m$ of the $\mathrm{S}$ domains defined as the magnetic moment of an individual $\mathrm{S}$ domain divided by the volume of this domain. (We remind that the magnetization of the $\mathrm{S}$ phase is undefined, but the magnetic moment is a well defined quantity [13].) As soon as the surface effects are neglected, $m$ is uniform and the average magnetization $\bar{m}$ over the sample is equal to $\rho_{s} m$. Therefore the field strength $H^{(i)}$ inside the $\mathrm{N}$ laminae is

$$
H^{(i)}=H-4 \pi \eta \bar{m}=H-4 \pi \eta \rho_{s} m,
$$

where $4 \pi \eta \rho_{s} m$ is the demagnetizing field.

Applying the Meissner condition to the $\mathrm{S}$ domains ( $B=$ $\left.H^{(i)}+4 \pi m=0\right)$, one obtains

$$
m=-\frac{H}{4 \pi\left(1-\eta \rho_{s}\right)} .
$$

Therefore the bulk magnetic energy $E_{m b}$ is (see Ref. [6] for details)

$$
E_{m b}=-V_{s} \int m d H=\frac{\rho_{s}}{1-\eta \rho_{s}} \frac{H^{2}}{8 \pi} V .
$$

In our model the sample is an infinite slab in a tilted field, implying that the demagnetizing factors for the parallel $\left(H_{\|}\right)$ and the perpendicular $\left(H_{\perp}\right)$ components of the applied field are $\eta_{\|}=0$ and $\eta_{\perp}=1$, respectively. Therefore the magnetic energy caused by $H_{\|}$is

$$
E_{m b \|}=V\left(1-\rho_{n}\right) \frac{H_{\|}^{2}}{8 \pi}=-V \rho_{n} \frac{H_{\|}^{2}}{8 \pi}+V \frac{H_{\|}^{2}}{8 \pi} .
$$

Comparing this expression with Eq. (3) in Ref. [12], we see that in order to calculate $M_{\|}$the term $\left(H_{\|}^{2} / 8 \pi\right) V$ has to be added in the same way as it has been done in Eq. (2) above. This accounts for the appearance of this positive term in Eq. (11).

The magnetic energy originating from $H_{\perp}$ is

$$
E_{m b \perp}=V \frac{1-\rho_{n}}{\rho_{n}} \frac{H_{\perp}^{2}}{8 \pi}=V \frac{H_{\perp}^{2}}{8 \pi \rho_{n}}-V \frac{H_{\perp}^{2}}{8 \pi} .
$$

Comparing Eq. (A5) with Eq. (3) in Ref. [12], we see that, in order to calculate $M_{\perp}$, the term $\left(H_{\perp}^{2} / 8 \pi\right) V$ must be subtracted. This explains the negative sign of this term in Eq. (11).
[1] M. Tinkham, Introduction to Superconductivity (McGraw-Hill, New York, 1996).

[2] G. N. Rjabinin and L. W. Shubnikow, Nature (London) 134, 286 (1934).

[3] Per F. Dahl, Hist. Stud. Phys. Biol. Sci. 16, 1 (1986).
[4] D. Shoenberg, Superconductivity, 2nd ed. (Cambridge University Press, Cambridge, U.K., 1952).

[5] A. A. Abrikosov, Fundamentals of the Theory of Metals (Elsevier, Amsterdam, 1988).

[6] A. Fortini and E. Paumier, Phys. Rev. B 5, 1850 (1972). 
[7] E. H. Brandt, Phys. Rev. B 71, 014521 (2005).

[8] C. J. Gorter and H. Casimir, Physica 1, 306 (1934).

[9] F. London, Physica 3, 450 (1936).

[10] R. Peierls, Proc. R. Soc. London, Ser. A 155, 613 (1936).

[11] L. D. Landau, Zh. Eksp. Teor. Fiz. 7, 371 (1937) [Phys. Zs. Sowjet. 11, 129 (1937), Collected paper of L. D. Landau (Gordon and Breach, 1965), p. 217].

[12] V. Kozhevnikov, R. J. Wijngaarden, J. de Wit, and C. Van Haesendonck, Phys. Rev. B 89, 100503(R) (2014).

[13] L. D. Landau, E. M. Lifshitz, and L. P. Pitaevskii, Electrodynamics of Continuous Media, 2nd ed. (Elsevier, Amsterdam, 1984).

[14] P. G. de Gennes, Superconductivity of Metals and Alloys (Westview, Boulder, CO, 1966).

[15] J. D. Livingston and W. DeSorbo, in Superconductivity, edited by R. D. Parks (Dekker, New York, 1969), Vol. II, p. 1235.

[16] M. Desirant and D. Shoenberg, Proc. R. Soc. London, Ser. A 194, 63 (1948).

[17] In Ref. [12] it is shown that samples can be considered "thick" (for which $B$ in the $\mathrm{N}$ laminae and $H_{c i}$ become equal to $H_{c}$ ), if $\sqrt{\delta / d} \ll 1$ (see Ref. [12] for the physical meaning of this ratio). Since $\delta$ increases with temperature $T$ and is diverging at
$T \rightarrow T_{c}$, the minimum thickness of a sample to be considered as "thick" increases with temperature as well. Experimentally, for a $0.56 \mathrm{~mm}$ thick tin slab in the perpendicular field the reduction of $H_{c i}$ was easily observed already at $T=0.08 \mathrm{~K}$ [18]. Sharvin [Zh. Eksp. Teor. Fiz. 33, 1341 (1957)] noticed a change of $H_{c i}$ in his $2 \mathrm{~mm}$ thick tin sample in a field tilted by only $15^{\circ}$ from the sample surface. Therefore, in order to be able to neglect the surface effects, the sample thickness should be at least a few mm. For "weak" type-I superconductors such as $\mathrm{Pb}(\kappa \approx 0.6)$, this thickness can be somewhat smaller.

[18] V. S. Egorov, G. Solt, C. Baines, D. Herlach, and U. Zimmermann, Phys. Rev. B 64, 024524 (2001).

[19] A. J. Walton, Proc. R. Soc. London, Ser. A 289, 377 (1966).

[20] J. E. Sonier, Rep. Prog. Phys. 70, 1717 (2007).

[21] V. Kozhevnikov, A. Suter, H. Fritzsche, V. Gladilin, A. Volodin, T. Moorkens, M. Trekels, J. Cuppens, B. M. Wojek, T. Prokscha, E. Morenzoni, G. J. Nieuwenhuys, M. J. Van Bael, K. Temst, C. Van Haesendonck, and J. O. Indekeu, Phys. Rev. B 87, 104508 (2013).

[22] V. I. Marchenko, Zh. Eksp. Teor. Fiz. 71, 2194 (1976) [JETP 44, 1156 (1976)].

[23] L. D. Landau, Zh. Eksp. Teor. Fiz. 13, 377 (1943) [J. Phys. USSR 7, 99 (1943)]. 